\title{
Selecting Cloud Computing Service Provider with Fuzzy AHP
}

\author{
Neda Tanoumand \\ FENS \\ Sabanci University \\ Istanbul, Turkey \\ ntanoumand@sabanciuniv.edu
}

\author{
Dicle Yagmur Ozdemir \\ FENS \\ Sabanci University \\ Istanbul, Turkey \\ dozdemir@sabanciuniv.edu
}

\author{
Kemal Kilic \\ FENS \\ Sabanci University \\ Istanbul, Turkey \\ kkilic@sabanciuniv.edu
}

\author{
Faran Ahmed \\ FENS \\ Sabanci University \\ Istanbul, Turkey \\ ahmedfaran@sabanciuniv.edu
}

\begin{abstract}
With the growing demand for outsourcing the ICT section of enterprises, Cloud Computing service providers increased their popularity. Selecting the most appropriate provider for a demanding enterprise depends on many criteria that are based on the strategies, requirements, and resources of the enterprise. Since this problem is a kind of decision problem and depends on criteria of decisionmaker, it can be modeled as Multi-criteria Decision Making (MCDM) problem. In this research, a pilot case study is conducted in which the Cloud Computing service provider selection problem is modeled as a MCDM problem. For selecting the most appropriate provider, Fuzzy Extend Analysis is implemented in the case study.
\end{abstract}

Keywords- Cloud Computing, Multi-Criteria Decision Making (MCDM), Fuzzy Extend Analysis, Selection Problem

\section{INTRODUCTION}

In competitive business world, information and communication technology (ICT) departments have become one of the most critical components of an enterprise for its survival. It has become a focal point for every enterprise and therefore a sizeable budget is allocated for its establishment and enhancement [1]. Establishing and/or outsourcing required technologies are strategic decisions that must be taken based on enterprise's strategies, needs and facilities [2]. Some organizations may prefer to establish the required technology by themselves, while other may prefer outsourcing. Since outsourcing decreases the cost of management and maintenance of new technology, it enhances the productivity and flexibility in work [3]. In last decades, Cloud Computing (CC) service provider companies have played a crucial role in supplement of required technology for demanding enterprises [1, 4].

CC services are categorized in three main models: Infrastructure as a Service (IaaS), Platform as a Service (PaaS), Software as a Service (SaaS). The basic services provided by $\mathrm{CC}$ are IaaS services which contain Network, Data Storage, and Security Services [5]. PaaS model includes the IaaS services and offers an Operating System, Database Management, and Business Analytics tools. In other words, PaaS contains all infrastructure services, additionally it includes some framework for development of cloud based applications [6]. The most complicated and advance service provided by $\mathrm{CC}$ is SaaS model. This category includes all PaaS services and hosting the applications [7].

By increase in the popularity of outsourcing the required technology using Cloud services, $\mathrm{CC}$ has become an attractive business sector and consequently, the number of CC service provider companies have been increased [2]. Therefore, technology demanding enterprises have to be careful about choosing the appropriate service provider which fulfills their needs [1]. Decision makers in such an enterprise must take conflicting criteria into consideration and choose the most suited service provider based on them. Because of the complexity of the problem, there are a lot of cases that $\mathrm{CC}$ service selection problem becomes intractable. Consequently, enterprises cannot determine the most appropriate provider and make the best decision conveniently.

Multi-criteria Decision Making (MCDM) is a subdiscipline of operations research which can handle the decision problems that include conflicting criteria. It contains methods and techniques which help decision maker to simplify and solve decision problem despite of the existence of conflicting criteria. These methods are categorized into two groups: Compensatory methods and Outranking methods. For detail see [8].

It seems that MCDM techniques are appropriate tools to tackle CC provider selection problem that is confounding because of the conflicting criteria. Modeling the problem as MCDM and using MCDM techniques convert the complex problem to a tractable and easy to solve one [9].

In the literature, there are articles which propose a general decision making framework for an enterprise which wants to choose the best service provider. Some of these general frameworks consider the enterprise's feedback regarding the solution of the framework and adjust the solution afterwards [10, 11]. Beside general decision making frameworks, there are number of reviews $[12,13]$ on application of MCDM techniques for solving $\mathrm{CC}$ service provider selection problem. Among MCDM techniques, in Compensatory category, Analytical Hierarchy Process (AHP) [14] is the most popular method that has been implemented for the problem [4, 15]. After AHP, ELECTRE from Outranking methods is the most observed one in the literature. Additionally, there exist some publications that utilize more than one MCDM techniques (called hybrid version) [16, 17].

In the context of decision making problems, sometimes it is hard for decision makers to compare criteria and alternatives numerically. Instead of quantitative comparisons, it can be more appropriate to use qualitative ones. For this purpose, fuzzy set theory is initially proposed by Zadeh [18], and has been extensively utilized in decision making problems. This is the efficient way to tackle 
ambiguity and vagueness of human judgement [19] and for this reason researchers have attempted to apply fuzzy version of MCDM techniques in Cloud Computing decision problems recently $[20,21]$. However, the number of articles which apply Fuzzy MCDM techniques in CC problems, especially $\mathrm{CC}$ service provider selection problem, is limited and requires more consideration. To fill this gap, in this study, Fuzzy AHP (FAHP) is implemented to solve a case of a $\mathrm{CC}$ service provider selection problem.

In this study, Sabanci University is considered as a demanding enterprise for IaaS. There is an expert in Computer Science department who is aware of requirements, technological basis, and the budget of the university. The expert knows the criteria and providers, and he can perform a pairwise comparison. However, existence of conflicting criteria makes the problem more complex that the expert cannot come up with the best decision. Through hierarchical steps of AHP, the expert analyzes and lists the requirements, criteria, and budget of the university. Additionally, fuzzy version makes comparison steps of AHP more convenient for the decision maker. Therefore, modeling this problem as MCDM and using FAHP technique lead to make a better decision. The criteria used in this study are obtained from the expert and based on the university's demands and strategies. FAHP algorithm, namely, Fuzzy Extent Analysis (FEA) is implemented in order to weight the criteria and rank the alternatives.

The rest of the paper is as follows: in section II a brief review of the Fuzzy AHP is provided, section III discusses the research methodology and we provide main results of our study in section IV. In the final section conclusion and future research areas are discussed.

\section{RESEARCH BACKGROUND}

Over the past several years, AHP process has evolved with particular focus on the choice of scale to utilize in order to accurately elicit human judgments from phrases such as "Moderate Importance", "Strong Importance" etc. Although the original scale proposed by Saaty [14] is still the most preferred scale, there are multiple disputes reported in the literature whether this scale can accurately transform human judgments into numbers. In order to address this issue, various scales have been proposed in the literature; however, the inherent uncertainty in the human judgments while providing comparison ratios is not sufficiently reflected in any of these proposed scales since they are all composed of crisp numbers. Disregarding this vagueness of the human behavior in the decision-making process could lead to wrong decisions [22] and thus fuzzy set theory [18] is extensively incorporated in the domain of AHP with a particular focus on accurately representing human judgments and perceptions along with the natural vagueness and uncertainty.

In this research, we utilize the fuzzy extension of AHP (FAHP) in which human judgments are elicited in the form of fuzzy numbers. The core of any FAHP model like traditional AHP is to accurately calculate weights from the comparison matrices which are composed of fuzzy numbers. Due to the intricacies associated with the fuzzy arithmetic, calculating weights from fuzzy comparison matrices is not straightforward and has been the focus of research over the past many years with various FAHP models proposed to calculate weights. Review of the existing literature shows that Logarithmic Least Square Method (LLSM) [23], Fuzzy Extent Analysis (FEA) [2, 24] and Buckley`s Geometric Mean method $[25,26]$ are among the most popular algorithms utilized by the researchers [25]. Fuzzy Extent Analysis is one of the most frequently used model by researchers in their decision-making problem. Therefore, in this study, we utilize the concept of Fuzzy Extent Analysis (FEA) [27] in order to implement Fuzzy AHP to elicit weights for each criterion. Pairwise comparisons provided by the experts are recorded in the form of fuzzy numbers and hence pairwise comparison matrices are composed of fuzzy numbers. FEA processes these fuzzy comparison matrices and extracts crisp weights from each matrix. This approach is further explained as follows:

Provided, $X=\left\{x_{1}, x_{2}, \ldots, x_{n}\right\}$ is an object set and $G=$ $\left\{g_{1}, g_{2}, \ldots, g_{n}\right\}$ is a goal set, then for each object, extent analysis for each goal $\mathrm{g}_{\mathrm{i}}$ is conducted to calculate value of fuzzy synthetic extent with respect to the $\mathrm{i}^{\text {th }}$ object. The same is mathematically illustrated as follows:

$$
\begin{array}{ll} 
& S_{i}=\sum_{j=1}^{m} M_{g_{i}}^{j} \otimes\left[\sum_{i=1}^{n} \sum_{j=1}^{m} M_{g_{i}}^{j}\right]^{-1} \\
\text { where } & \sum_{j=1}^{m} M_{g_{i}}^{j}=\left(\sum_{j=1}^{m} l_{j}, \sum_{j=1}^{m} m_{j}, \sum_{j=1}^{m} u_{j}\right)
\end{array}
$$

Note that when the comparison matrix is composed of crisp numbers, above operations on the comparison matrix would yield the desired weight vector. However, as matrices are composed of fuzzy numbers, the result of these operations would be a fuzzy triangular weight and in the decision-making process, while choosing the best alternative, we need to determine a crisp weight from these fuzzy triangular weights.

Various different approaches can be considered in order to convert fuzzy triangular weights into crisp weights. A naive approach would be just using the means or centroids of the triangular fuzzy number. However, extra care should be taken in the ordering of fuzzy number due to its unique structure. In FEA approach, this ordering is conducted through the concept of comparison of fuzzy numbers i.e. for each fuzzy number (fuzzy weight), a pairwise comparison with the other fuzzy numbers (fuzzy weights) is conducted and the degree of possibility of being greater is obtained as follows: (the same is illustrated in Figure I.)

$$
\begin{gathered}
V\left(M_{2} \geq M_{1}\right)=\operatorname{hgt}\left(M_{1} \cap M_{2}\right)=\mu_{M_{2}}(d)= \\
\left\{\begin{array}{cc}
1, & \text { if } m_{2} \geq m_{1} \\
0, & \text { if } l_{1} \geq u_{2} \\
\frac{l_{1}-u_{2}}{\left(m_{2}-u_{2}\right)-\left(m_{1}-l_{1}\right)}, & \text { otherwise. }
\end{array}\right.
\end{gathered}
$$

Minimum of these possibilities are considered as the overall score for each criterion $i$ i.e. degree of possibility for a convex fuzzy number to be greater than $\mathrm{k}$ convex fuzzy numbers is given by: 


$$
\begin{gathered}
V\left(M \geq M_{1}, M_{2}, \ldots, M_{k}\right)=V\left[\left(M \geq M_{1}\right)\right. \text { and } \\
\left.\left(M \geq M_{2}\right), \ldots,\left(M \geq M_{k}\right)\right] \\
=\min V\left(M \geq M_{i}\right), \quad i=1,2, \ldots, k
\end{gathered}
$$

In the final step, these scores are normalized and the corresponding normalized scores are used as the weight vector of the criteria.

\section{MethodOLOGY}

CC service provider selection problem can be considered as a multi-criteria decision making problem, thus MCDM techniques can be used for this purpose. Among these techniques, Fuzzy Analytical Hierarchy Process (FAHP) is considered as one of the most appropriate technique for the CC service provider selection problem. Since, the underlying hierarchical structure of FAHP models the problem properly and makes it more tractable. Additionally, fuzzy scales provide a better representation of the vagueness in the human judgment based on comparisons of the criteria and the alternatives for service demanding enterprises.

In the literature, $\mathrm{CC}$ service provider selection problem usually handled independent from the service category and generic models are presented. However, certain criteria are peculiar to a particular service category so such a generalization is misleading. In this paper, the focus is on IaaS service provider selection problem and obtained criteria are particular to this category. FAHP algorithm is implemented in order to choose the best provider for the enterprise. This approach involves multiple consecutive steps, namely, criteria selection, weighting the criteria, determination of the $\mathrm{CC}$ service provider alternatives, and finally, overall scoring of the providers.

\section{A. Criteria selection}

To implement an MCDM techniques in a service provider selection problem, one of the things that should be done is determining the criteria that will be used in the analysis. Even though the significance of each criteria for different decision makers might be different and depend mostly to the strategy and the requirements of the enterprise, the criteria themselves mostly overlap among the applications. However, different service models have different criteria. Therefore, for cloud computing service provider selection problem, a service model based list of criteria should be identified before the implementation. There are so many criteria that have been defined and

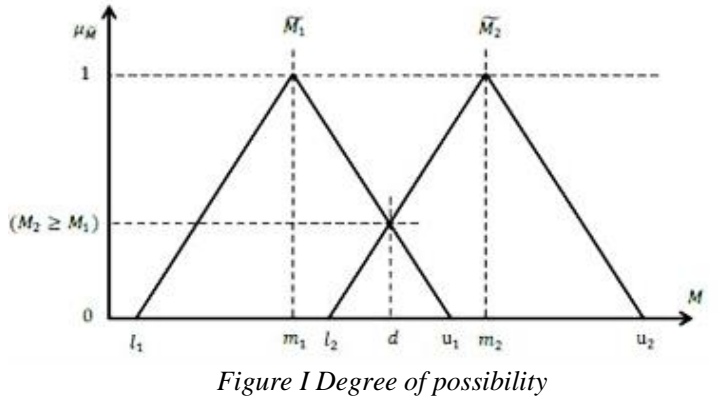

considered in the literature. The subjective criteria are obtained from the expert which are based on university's requirements and cross checked by the similar studies to confirm the validity $[11,28,29]$. As a result, for the IaaS provider selection case study the following six criteria are used:

- Acquisition and transaction cost

- Availability

- Storage capacity

- $\mathrm{CPU}$

- Performance

- Security

\section{B. Weighting criteria}

In this study, criteria weighting is done by using fuzzy scales. To this end, a questionnaire that compatible with fuzzy scales have to be prepared and given to the related expert for pairwise comparison of the criteria. The linguistic scales used in questionnaire and their corresponding fuzzy scales are presented in Table I. After getting the comparison data from the expert, numerical matrices are prepared. Finally, by using numerical matrices, a weight for each criterion is calculated using FEA.

\section{Specification of CC service providers}

In this step, the $\mathrm{CC}$ service provider alternatives are determined. The CC service providers are chosen among the well-known companies and the list is finalized after consulting the expert. As a result, five service providers have been selected, namely, Amazon AWS, Windows Azure, Google Compute Engine, Rackspace Open Cloud and IBM SmartCloud Enterprise. Various specifications of these alternatives in terms of the criteria are determined from the open sources and provided to the expert before the comparison stage.

\section{Scoring CC service providers}

For achieving FAHP score of every specified provider, it is needed to compare the companies in pairs in terms of each criterion. To this end, a questionnaire is prepared to be filled by the expert in the demanding enterprise. The questionnaire must be prepared to be compatible with FAHP implementation on the selection problem so that the comparison scales will be consistent with the FAHP scales. Linguistic scales and their corresponding triangular fuzzy scale used in this work have been selected as shown in Table I.

After obtaining comparison data from the expert, linguistic scales are converted into numerical scales based on Table I, so that numerical comparison matrix is prepared. Next, FEA [24] is implemented for score calculation. The calculations are done in MATLAB. For IaaS provider selection problem, matrices are created with six criteria for five companies. The achieved results from the implementation are discussed in the following section. 


\section{RESULTS AND DiSCUSSION}

In this study, we considered five $\mathrm{CC}$ service provider alternatives for IaaS provider selection problem, namely Amazon, Windows, Google, Rackspace and IBM. These alternatives were analyzed based on six criteria namely acquisition and transaction cost, availability, storage capacity, CPU, performance, and security. Pairwise comparisons were sought from a field expert in order to compare all available criteria as well as alternatives depending on each criterion. Based on these comparisons, fuzzy comparison matrices were constructed and a FAHP algorithm namely Fuzzy Extent Analysis [24] was utilized to elicit weights from fuzzy comparison matrices for each criterion as well as alternative. These results are demonstrated in Table II and III.

Note that one of the criticisms of Fuzzy Extent Analysis is based on the fact that in certain instances, due to structure of fuzzy number comparison, it can provide zero as a weight elicited from the matrices. We observe the same phenomenon while comparing all criteria where "acquisition and transaction cost" was given a zero weight. However, this does not change the overall rank of the alternatives. The outcomes of FEA method illustrates that security is the most important criteria for our case study and Amazon is the most appropriate CC service provider for the university with respect to its requirements. Note that the results might differ for other enterprises, since

Table I Linguistic scales and corresponding Triangular fuzzy scales [11]

\begin{tabular}{|l|l|}
\hline Linguistic scale & Triangular fuzzy scale \\
\hline Just equal & $(1,1,1)$ \\
Equally important & $(1 / 2,1,3 / 2)$ \\
Weakly more important & $(1,3 / 2,2)$ \\
Strongly more important & $(3 / 2,2,5 / 2)$ \\
Very strongly more important & $(2,5 / 2,3)$ \\
Absolutely more important & $(5 / 2,3,7 / 2)$ \\
\hline
\end{tabular}

Table II Weight calculation for each criterion using Chang's method

\begin{tabular}{|l|c|}
\hline Criteria & Criteria weights \\
\hline $\begin{array}{l}\text { Acquisition and } \\
\text { transaction cost }\end{array}$ & 0.000 \\
\hline Availability & 0.104 \\
\hline Storage Capacity & 0.050 \\
\hline CPU & 0.271 \\
\hline Performance & 0.214 \\
\hline Security & 0.361 \\
\hline
\end{tabular}

Table III Scores obtained for each alternative using Chang's method

\begin{tabular}{|l|c|}
\hline \multicolumn{2}{|c|}{ Alternative Weights } \\
\hline Amazon & 0.385 \\
\hline Windows & 0.218 \\
\hline Google & 0.216 \\
\hline Rackspace & 0.131 \\
\hline IBM & 0.050 \\
\hline
\end{tabular}

pairwise comparisons of criteria and alternatives are enterprise-dependent.

\section{CONCLUSION}

With increasing demand of enterprises for outsourcing their ICT section along with the growth of popularity of Cloud Computing services, some tools are required for matching demands with suppliers. To this end, in this study, CC service provider selection problem have been modeled as MCDM problem. Then, Fuzzy AHP technique has been implemented via FEA method. Ultimately, the most suitable supplier has been specified for the inspected enterprise.

Since the selection problem can be modeled as demanding-supplier problem, for further studies, other appropriate tools can be implemented for solving it. Additionally, according to the rapid increase in CC service demands, development of professional software is required in order to undertake the selection problem based on specific criteria.

\section{ACKNOWLEDGMENT}

We thank Asst. Prof. Kamer Kaya from Sabanci University who assisted us with his wisdom and expertise during the course of the research.

\section{REFERENCES}

[1] H.-K. Kwon and K.-K. Seo, "A decision-making model to choose a cloud service using Fuzzy AHP," Advanced Science and Technology Letters, vol. 35, pp. 93-96, 2013.

[2] P. Costa, "Evaluating Cloud Services using Multicriteria Decision Analysis," ed, 2013.

[3] S. Wibowo and H. Deng, "Evaluating the Performance of Cloud Services: A Fuzzy Multicriteria Group Decision Making Approach," in Computer, Consumer and Control (IS3C), 2016 International Symposium on, 2016, pp. 327-332.

[4] C. Yiming and Z. Yiwei, "SaaS Vendor Selection Basing on Analytic Hierarchy Process," in Computational Sciences and Optimization (CSO), 2011 Fourth International Joint Conference on, 2011, pp. 511-515.

[5] Z. ur Rehman, F. K. Hussain, and O. K. Hussain, "Towards multi-criteria cloud service selection," in Innovative Mobile and Internet Services in Ubiquitous Computing (IMIS), 2011 Fifth International Conference on, 2011, pp. 44-48.

[6] M. Abourezq and A. Idrissi, "Introduction of an outranking method in the Cloud computing research and Selection System based on the Skyline," in 2014 IEEE Eighth International Conference on Research Challenges in Information Science (RCIS), 2014, pp. 1-12.

[7] M. Godse and S. Mulik, "An approach for selecting software-as-a-service (SaaS) product," 
in 2009 IEEE International Conference on Cloud Computing, 2009, pp. 155-158.

[8] S. Greco, J. Figueira, and M. Ehrgott, "Multiple criteria decision analysis," Springer's International series, 2005.

[9] S. K. Garg, S. Versteeg, and R. Buyya, "Smicloud: A framework for comparing and ranking cloud services," in Utility and Cloud Computing (UCC), 2011 Fourth IEEE International Conference on, 2011, pp. 210-218.

[10] L. Qu, Y. Wang, and M. A. Orgun, "Cloud service selection based on the aggregation of user feedback and quantitative performance assessment," in Services Computing (SCC), 2013 IEEE International Conference on, 2013, pp. 152159.

[11] R. Garg, B. Naudts, S. Verbrugge, and B. Stiller, "Modeling legal and regulative requirements for ranking alternatives of cloud-based services," in Requirements Engineering and Law (RELAW), 2015 IEEE Eighth International Workshop on, 2015, pp. 25-32.

[12] M. Whaiduzzaman, A. Gani, N. B. Anuar, M. Shiraz, M. N. Haque, and I. T. Haque, "Cloud service selection using multicriteria decision analysis," The Scientific World Journal, vol. 2014, 2014.

[13] P. Costa, J. P. Santos, and M. M. da Silva, "Evaluation criteria for cloud services," in 2013 IEEE Sixth International Conference on Cloud Computing, 2013, pp. 598-605.

[14] T. L. Saaty, The Analytic Hierarchy Process: Planning, Priority Setting, Resource Allocation: McGraw-Hill, 1980.

[15] Y.-C. Lee and T. N. Hanh, "A Study on Decision Making Factors of Cloud Computing Adoption Using BCOR Approach," Journal of the Korea society of IT services, vol. 11, pp. 155-171, 2012.

[16] S. Liu, F. T. Chan, and W. Ran, "Decision making for the selection of cloud vendor: An improved approach under group decision-making with integrated weights and objective/subjective attributes," Expert Systems with Applications, vol. 55, pp. 37-47, 2016.

[17] H.-K. Kwon and K.-K. Seo, "A Fuzzy AHP based Multi-criteria Decision-making Model to Select a Cloud Service," International Journal of Smart Home, vol. 8, pp. 175-180, 2014.

[18] L. A. Zadeh, "Fuzzy sets," Information and Control, vol. 8, pp. 338-353, 6// 1965.

[19] T.-C. Chu and Y. Lin, "An extension to fuzzy MCDM," Computers \& Mathematics with Applications, vol. 57, pp. 445-454, 2// 2009.

[20] I. Patiniotakis, S. Rizou, Y. Verginadis, and G. Mentzas, "Managing imprecise criteria in cloud service ranking with a fuzzy multi-criteria decision making method," in European
Conference on Service-Oriented and Cloud Computing, 2013, pp. 34-48.

[21] N. Badie, A. R. C. Hussin, and A. H. Lashkari, "Cloud Computing Data Center Adoption Factors Validity By Fuzzy AHP," International Journal of Computational Intelligence Systems, vol. 8, pp. 854-873, 2015.

[22] S.-H. Tsaur, T.-Y. Chang, and C.-H. Yen, "The evaluation of airline service quality by fuzzy MCDM," Tourism management, vol. 23, pp. 107115, 2002.

[23] P. J. M. van Laarhoven and W. Pedrycz, "A fuzzy extension of Saaty's priority theory," Fuzzy Sets and Systems, vol. 11, pp. 229-241, // 1983.

[24] D.-Y. Chang, "Applications of the extent analysis method on fuzzy AHP," European Journal of Operational Research, vol. 95, pp. 649-655, 1996/12/20 1996.

[25] J. J. Buckley, "Fuzzy hierarchical analysis," Fuzzy Sets and Systems, vol. 17, pp. 233-247, 12// 1985.

[26] S. K. Garg, S. Versteeg, and R. Buyya, "A framework for ranking of cloud computing services," Future Generation Computer Systems, vol. 29, pp. 1012-1023, 2013.

[28] Z. ur Rehman, O. K. Hussain, and F. K. Hussain, "Iaas cloud selection using MCDM methods," in e-Business Engineering (ICEBE), 2012 IEEE Ninth International Conference on, 2012, pp. 246251.

[29] Z. ur Rehman, O. K. Hussain, and F. K. Hussain, "Multi-criteria IaaS service selection based on QoS history," in Advanced Information Networking and Applications (AINA), 2013 IEEE 27th International Conference on, 2013, pp. 1129-1135. 